\title{
VIRTUAL TOUR IN THE SUNKEN "VILLA CON INGRESSO A PROTIRO" WITHIN THE UNDERWATER ARCHAEOLOGICAL PARK OF BAIAE
}

\author{
F. Bruno ${ }^{1,2, *}$, A. Lagudi $^{2}$, L. Barbieri ${ }^{1}$, M. Cozza ${ }^{2}$, A. Cozza ${ }^{2}$, R. Peluso ${ }^{2}$, B. Davidde Petriaggi ${ }^{3}$, R. Petriaggi ${ }^{3}$, S. Rizvic $^{4}$, D. \\ Skarlatos 5 \\ ${ }^{1}$ Department of Mechanical, Energy and Management Engineering (DIMEG), University of Calabria, 87036 Rende (CS), Italy \\ 2 3DResearch s.r.1., 87036 Rende (CS), Italy \\ ${ }^{3}$ Istituto Superiore per la Conservazione ed il Restauro, 00153 Roma, Italy \\ ${ }^{4}$ Faculty of Electrical Engineering, Department of Computer Science, University of Sarajevo, 71000 Sarajevo, Bosnia and \\ Herzegovina \\ ${ }^{5}$ Department of Civil Engineering and Geomatics, Cyprus University of Technology, 3036 Limassol, Cyprus
}

Commission II, WG II/9

KEY WORDS: Virtual Reality, Virtual Heritage, 3D hypothetical reconstruction, digital storytelling, Virtual Diving.

\begin{abstract}
:
The paper presents the application of some Virtual Reality technologies developed in the Horizon 2020 i-MARECulture project to the case study of the sunken "Villa con ingresso a protiro", dated around the II century AD, and located in the Marine Protected Area - Underwater Park of Baiae (Naples).

The i-MARECulture project (www.imareculture.eu), in fact, aims to improve the public awareness about the underwater cultural heritage by developing new tool and techniques that take advantage of the virtual reality technologies to allow the general public to explore the archaeological remains outside of the submerged environment.

To this end, the paper details the techniques and methods adopted for the development of an immersive virtual tour that allow users to explore, through a storytelling experience, a virtual replica and a 3D hypothetical reconstruction of the complex of the "Villa con ingresso a protiro".
\end{abstract}

\section{INTRODUCTION}

The Underwater Cultural Heritage $(\mathrm{UCH})$ represents a vast historical and scientific resource that, in the vast majority of cases, is not accessible to the general public because of the environmental constraints. In fact, only expert persons, with a diving license, can overcome the difficulties imposed by the environment and depth to enjoy the submerged cultural treasures.

Modern technologies offer various solutions for enhancing perception, awareness, and knowledge of cultural and natural heritage and thus contribute to its efficient conservation and sustainable promotion. In particular, computer graphics techniques have demonstrated to be effective in increasing the value and the exploitability of the cultural heritage especially when archaeological sites and artefacts are not accessible because of physical and spatial limitations (the museum is far away, the artefact belongs to a traveling collection or, even worse, is locked away in storage's museum) or human body limits (tourists could present physical conditions that are inadequate to visit a specific archaeological site). Therefore, it's quite evident how digital technologies (Virtual Museums, Virtual Guides and Virtual Reconstruction of Cultural Heritage) provide a unique opportunity for digital accessibility to anyone interested in having a better grasp of underwater sites and maritime archaeology.

\footnotetext{
* Corresponding author-fabio.bruno@unical.it
}

Among the digital technologies, Virtual Reality (VR) has proven its effectiveness in increasing the value of cultural heritage and in the last years it has been efficiently applied for the visualization and exploitation of the UCH (Chapman et al., 2006; Liarokapis et al., 2017; Bruno et al., 2016, 2018). In fact, VR technology can be efficiently applied to this field in order to make underwater archaeological sites the most accessible to the general public without any constraint given by distance, depth or time.

In this context, the Horizon 2020 i-MARECULTURE (Advanced VR, iMmersive Serious Games and Augmented REality as Tools to Raise Awareness and Access to European Underwater CULTURal heritage) project takes advantage of the VR technology for promoting and improving the public awareness about the UCH (iMareCulture, 2019; Bruno et al., 2017; Skarlatos et al., 2016).

To this end, the paper presents a virtual diving system that combines the advantages offered by the recent advances in VR technology with the newest $3 \mathrm{D}$ reconstruction techniques to create virtual tours for the exploitation of the UCH. In particular, the paper focuses on the virtual tour of the complex of the "Villa con ingresso a protiro" dated to the first half of the II century $\mathrm{AD}$, and located in the Marine Protected Area Underwater Park of Baiae (Naples).

The paper is organized as follows. Section 2 describes the archaeological context. Section 3 provides an overview of the VR system and application. The development of the interactive 
virtual scenario is detailed in Section 4, while user experience and interaction are described in Section 4. Finally, the conclusions are presented in Section 5.

\section{ARCHAEOLOGICAL CONTEXT}

Baiae was a famous coastal settlement in the volcanic Phlegraean Fields, much appreciates by the Roman aristocracy since the Late Republic (II-I century BC). The thermal waters and the climate favored the development of a monumental and residential architecture which was improved by the Emperors during the following centuries (I-IV AD). At the end of the fourth century, beginning of the fifth century $\mathrm{AD}$, several ground movements (bradyseism) caused the sinking of a portion of the city below the sea level. Nowadays, most of the buildings of the city lie on the bottom of the sea covering an area of about 177 hectares that since 2002 has been declared a Marine Protected Area. The scuba divers can visit several underwater paths: the Nymphaeum of Claudius lying off Punta Epitaffio with the copies of the original statues, now exposed in the Phlegraean Fields Museum; the remains of the Villa dei Pisoni, with the spectacular architectural changes due to emperor Hadrian; the remains of houses and storehouses of Portus Iulius; the "Villa con ingresso a protiro" siding a road along which there were thermae, tabernae, and other buildings.

About the "Villa con ingresso a protiro" (fig.1) it has two clearly defined areas, the residential quarters with the garden (the square area in the centre of figure 1) and the baths on the left side of the garden.

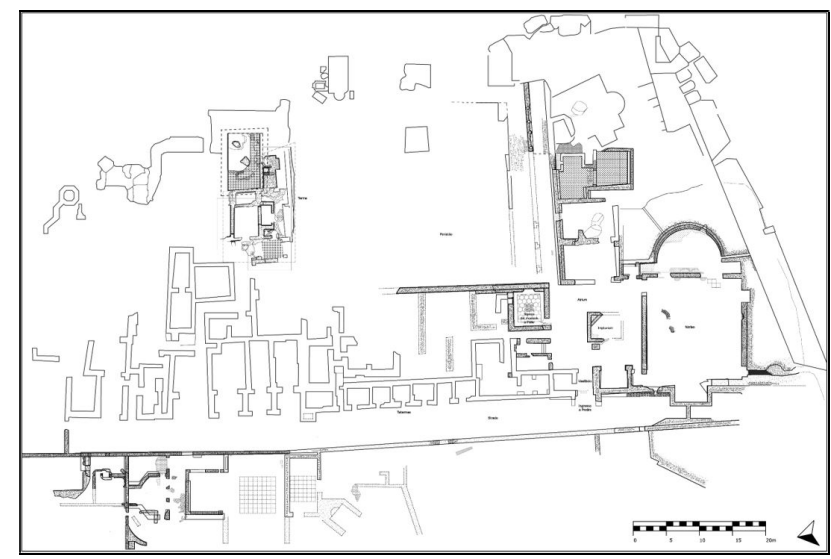

Figure 1. Archaeological map of "Villa con ingresso a protiro" (C) ISCR

Two red-plastered masonry benches - framed by stucco pilasters - marked out the threshold along the road. The vestibule, with doorways to the 'ostiarius quarters' (gatekeeper's lodge), led to the atrium with impluvium in the center, whose walls were decorated in the lower part with marble slabs. Also, the walls of the adjacent rooms were decorated with marble, many of which had mosaic flooring. A mosaic floor in black and white tesserae with geometrical motifs (hexagons and pseudo-emblemas with circles and peltae) is positioned in a room at the north-eastern corner of the atrium.

This mosaic, classifies by ISCR as n. VIP 19, lies inside a rectangular area measuring 4.77 metres by 5.55 metres in the northwestern part of the Villa. It consists of a background of white tesserae, with the ornamental motifs bordered by a double line of black tesserae. The principal geometrical design is a series of hexagons covering the whole floor, with a small square of four black tesserae at the centre of each hexagon. Black tesserae also make up the double border at the edges. The central section is a pseudo-emblema consisting of four circles inside a large square. Each circle contains four peltae and a central quincunx (the five-spot motif on six-sided dice). There are three equidistant solid black equilateral triangles outside the circles on each of the inside edges of the square. The point of each pelta terminates in a small flower with three heart-shaped petals separated by a central dot. The central space between the four circles contains a small square enclosing a cruciform floral motif with a central dot. The floral motif has lobed petals with arrowhead tips. The mosaic dates back to the second century AD.

A large hall with a 10.37 metres wide apse lies to the South of the atrium. This was probably not part of the original design but at the moment it is impossible to establish the date of construction and its relation with it. Its general appearance, and in particular the sumptuous large-scale marble panelling, is reminiscent of the grand halls of the Late-Imperial domus of Ostia. The "Villa con ingresso a protiro" was restored for the first time in 2003 thanks to the "Restoring Underwater" Project that led to the restoration of a room with a white mosaic floor measuring around 4.5 metres long and 2.5 metres wide (classified as $\mathrm{n} .14$ by ISCR) (Petriaggi and Mancinelli, 2004). Then, on 2010 a thermal room (caldarium) was restored under the direction of Roberto Petriaggi. In 2012 another campaign of 3D documentation (Davidde Petriaggi, 2017) and conservation has been executed under the direction of Barbara Davidde Petriaggi, with the restoration of some sectors of the archaeological complex and in particular the room paved with the black and white mosaic floor (fig.2).
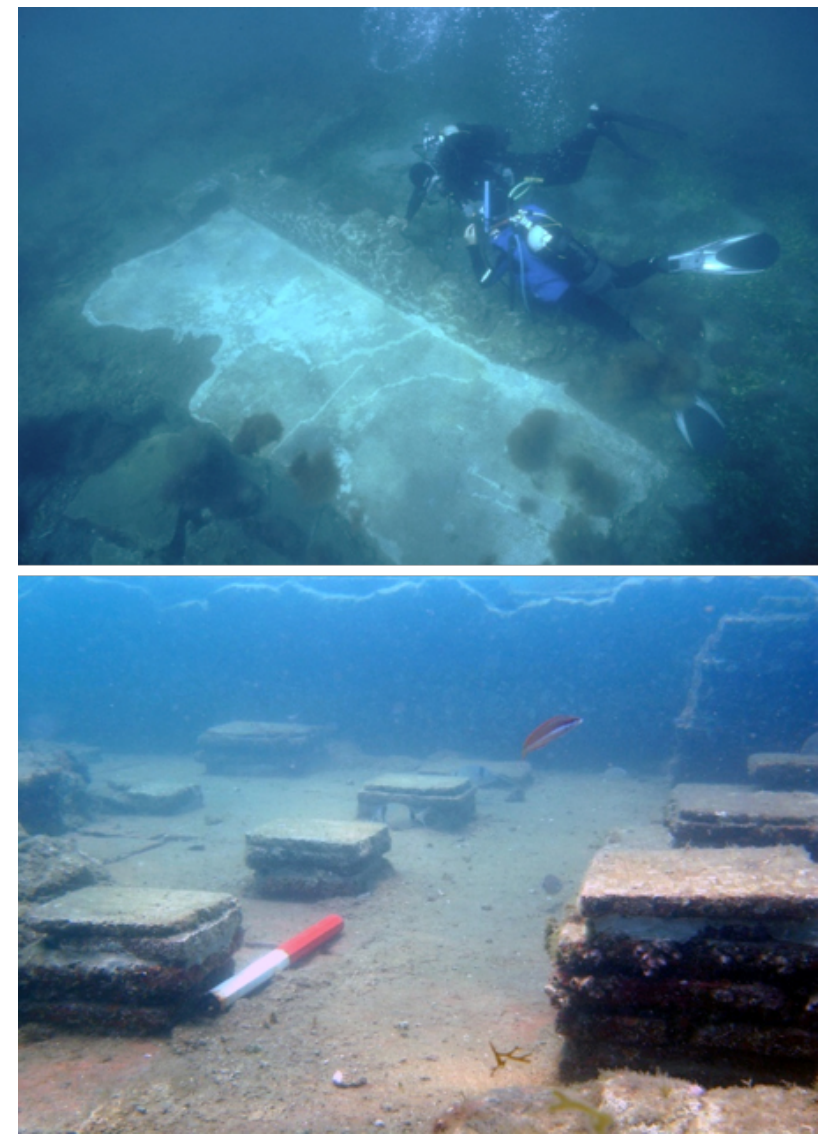

Figure 2. ISCR restoration activities in the "Villa con ingresso a protiro" (C) ISCR 


\section{VR DIVING SYSTEM OVERVIEW}

The software architecture (fig.2) of the virtual diving system consists of four elements: a Database (DB), a Web Service, a Scene Editor Module, and the VR Application.

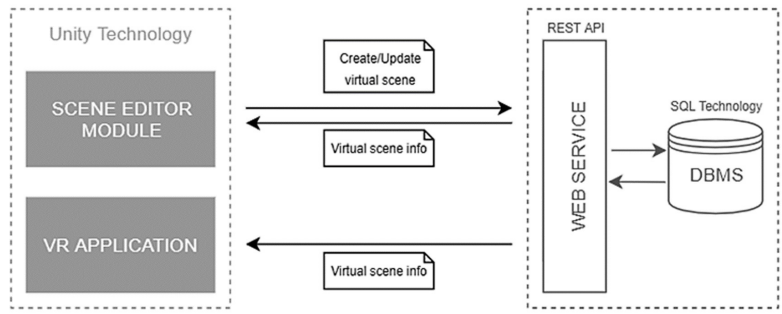

Figure 2. Software architecture of the VR diving system

The DB manages all of the data of the virtual scenes. The Web Service provides a set of functions for the mutual communication between the DB and the other software modules. The Scene Editor Module allows creating the virtual scene by integrating $3 \mathrm{D}$ objects (the $3 \mathrm{D}$ reconstruction of the underwater site, 3D models of flora and fauna typical of the specific marine ecosystem, etc.) and multimedia information (structured as Point of Interests, POIs) stored in the DB. Finally, the VR Application is the module where the logic of the virtual scenario is implemented by defining the physics and behaviours of the elements that belong to the virtual environment. Moreover, it allows performing the exploration within the virtual scenario according to the user inputs.

\subsection{Scene Editor Module}

As mentioned above, the Scene Editor Module allows creating virtual scenes by adding the POIs stored in the DB to the $3 \mathrm{D}$ model of the underwater site. The module is composed of the Scene Editor component (fig.3) and a Unity project, used to create an Asset Bundle (fig.4). The Scene Editor component contains all the business logic for the scene creation (communication with the Web Service: load and save scenes, add POIs, create Scuba guide's route, etc.).

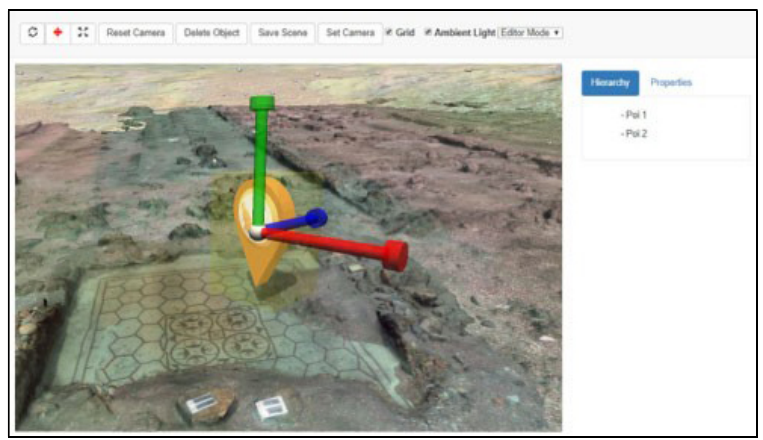

Figure 3. Scene Editor component for the creation of the underwater virtual scenario

The Unity project contains the 3D model of the underwater archaeological site that has been recorded by Gabriele Gomez de Ayala for ISCR in 2017. It has been enriched with the surrounding seafloor, flora and fauna, the terrestrial environment (coastline, etc.), and additional 3D models (boat, buoy, etc.). It is used to create an Asset Bundle that is saved in the DB and loaded in the Scene Editor component to create the virtual scenario. The Asset Bundle is an archive file containing platform-specific Assets (models, textures, prefabs, audio clips) that can be loaded at runtime in the application. The workflow to create the virtual scene is the following:

- the level designer creates the virtual environment using the Unity editor;

- the level designer builds the Asset Bundle containing the virtual environment of the specific underwater site;

- the editor adds all the information (virtual scenario, POIs contents) to the DB by means of the Web Service app

- the editor places the POIs within the virtual environment through the Scene Editor and creates the scuba guide route.

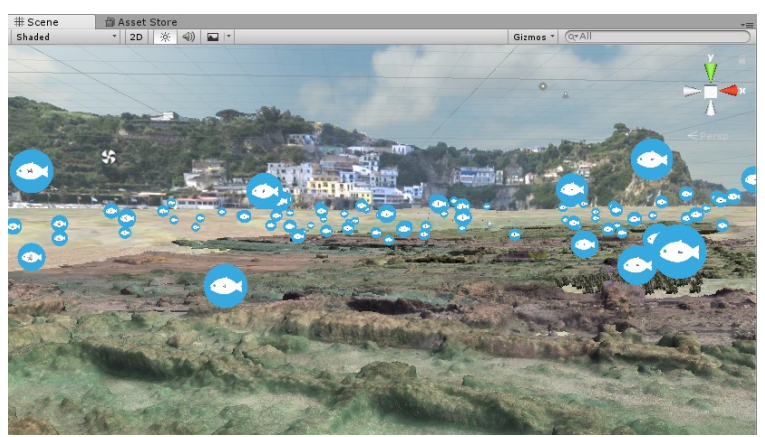

Figure 4. Unity project used to create the Asset Bundle of the underwater virtual scenario

\subsection{VR application}

The VR application is a virtual diving exhibit that allows users to explore and interact with the $3 \mathrm{D}$ reconstruction of the underwater site and receive historical and archaeological information about the submerged artefacts.

It is worth to point out that the VR application allows to dynamically load the scenario (namely, the underwater archaeological site scene). In this manner, it is possible to manage a unique application, with the same business logic, to handle different and various scenarios.

In order to achieve the best results in terms of user experience, the VR application can be exploited by means of the Head Mounted Display (HMD) technology. In fact, this technology provides to its users an immersive experience of the underwater archaeological site. The user wears a light-weight helmet that isolates him/her from the distractions of the actual physical environment and encompasses the entire field of view, including the peripheral space. It contains a high-resolution stereoscopic display; adjustable optics; an optical tracking system capable of tracking both the position and the orientation of user's head; and, usually, a stereo audio output.

The HMD is usually coupled with one or two wireless handheld controllers for a better experience in the VR environment. Each controller is equipped with several buttons, joystick, or touchpad as a means of human-computer interaction. They are tracked in terms of position and orientation in 3D space. The use of this configuration (HMD and a pair of tracked controllers) provides an immersive natural interaction with virtual worlds; the user can freely walk and look around, reach out their hands, and interact with virtual objects. In particular, the VR application has been developed in Unity for the HTC Vive VR headset. The HTC Vive features a resolution of $1080 \times 1200$ per eye, $90 \mathrm{~Hz}$ refresh rate, and a field of view of $110^{\circ}$. Furthermore, it comes bundled with two motion tracked controllers and laser-based tracking system called Lighthouse, 
providing six degrees of freedom (6-DOFs) tracking in an up to $4.5 \mathrm{~m} \times 4.5 \mathrm{~m}$ area with two beacons.

\section{INTERACTIVE VIRTUAL SCENARIO}

The interactive virtual scenario of the complex of the "Villa con ingresso a protiro" is created starting from the textured $3 \mathrm{D}$ model of the underwater archaeological area (Bruno et al., 2015; Mangeruga et al., 2018). Then, by means of the scene editor described in Section 3.1, the 3D reconstruction of the underwater area is populated with POIs in the form of 3D large head map tacks which color depends by the category they belong to, e.g., yellow (fig.5a) for the historical and archaeological information and green for biological ones.

In particular, multimedia data are assigned to each POI that consist in: audio files that provide archeological, historical and biological information; a 3D hypothetical reconstruction of the "Villa con ingresso a protiro", based on the integration of 3D data and historical analysis; and also 360-degree videos settled in the abovementioned hypothetical reconstruction scenario.
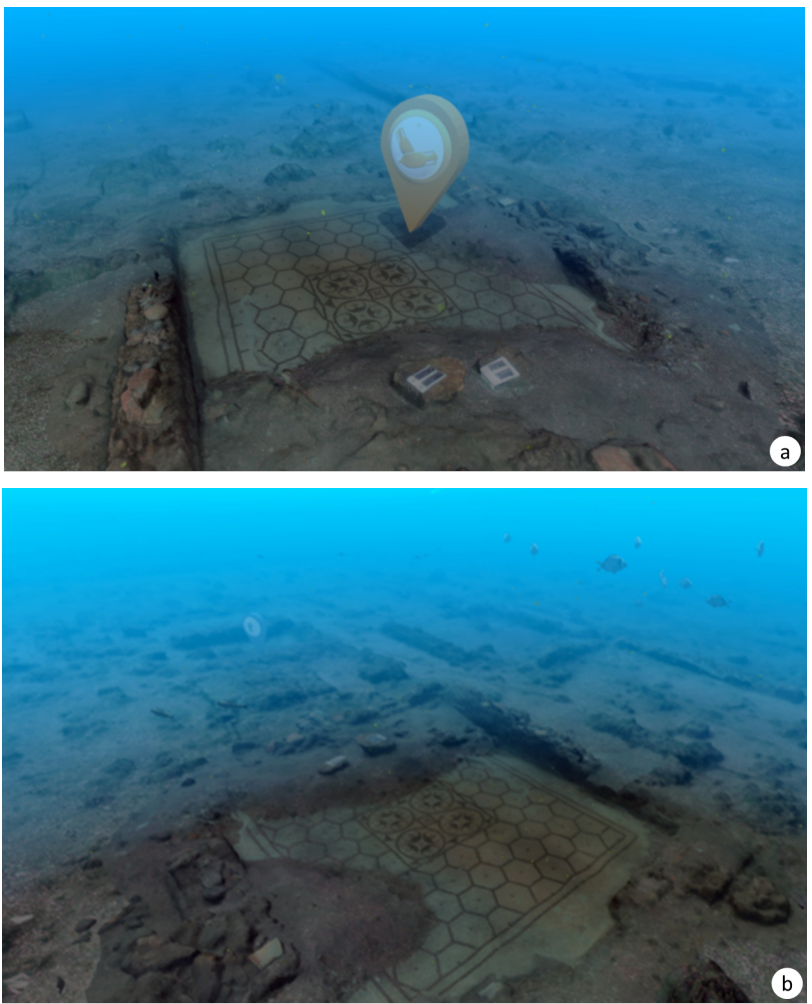

Figure 5. Adding a 3D object POI in the virtual scene (a); interactive virtual scene of sunken "Villa con ingresso a protiro" (b)

Furthermore, in order to enhance the realism of the virtual underwater environment, graphical effects and physically accurate simulations, such as light rays, refractions, fog, caustics, particles, and bubbles have been added to the scene. This has been also populated with 3D models of flora and fauna typical of the specific marine ecosystem (fig.5b). In particular, 3D models of fishes and schools of fish, typical of that site, are settled into the underwater environment and animated by means of artificial intelligence techniques. Moreover, the vegetation is reproduced employing texture effects that mimic the movements of the real plants.

\subsection{D hypothetical reconstruction}

About the abovementioned 3D hypothetical reconstruction of the "Villa con ingresso a protiro", this has been achieved by means of a theoretical and multidisciplinary scientific approach (Davidde Petriaggi et al., 2018) that exploits the high-resolution 3D data together with drawings and other historical and archaeological information to build a suggestive and consistent 3D digital reconstruction of the underwater architectures not anymore existing. In particular, the approach is based on an iterative feedback reconstruction process that involves several professional figures, such as 3D graphic designers, archaeologists, and art historians, for creating a scientifically sound virtual reconstruction of the site as it appeared in the past. The iterative process in fact, on the one hand, supports the archaeologists to focus their reasoning through a detailed visual representation better, and, on the other hand, helps the technical experts to avoid misleading details in the final virtual model.

The reconstruction process starts with the gathering of historical documentation, scientific literature and geometric data collected during the survey campaign. All these data (archaeological maps, illustrations, photos, Digital Terrain Model, etc.) are then analysed and put in relation by the experts involved in studies, and different interpretation hypotheses are investigated. The next step of the process consists of modelling the architectural remains and validating it by means an iterative critical revision. The process, in fact, is based on interleaving a phase of technical reconstruction with a strong critical revision in order to generate a feedback process, iterating the construction /correction loop as much as needed. Finally, to map the evolution of the virtual interpretation, several 3D layers are saved together with the final model, examined and approved by the scientific experts. The following figure (Fig.5) depicts different portions of the final 3D reconstruction, as it appeared in the past, of the complex of "Villa con ingresso a protiro".

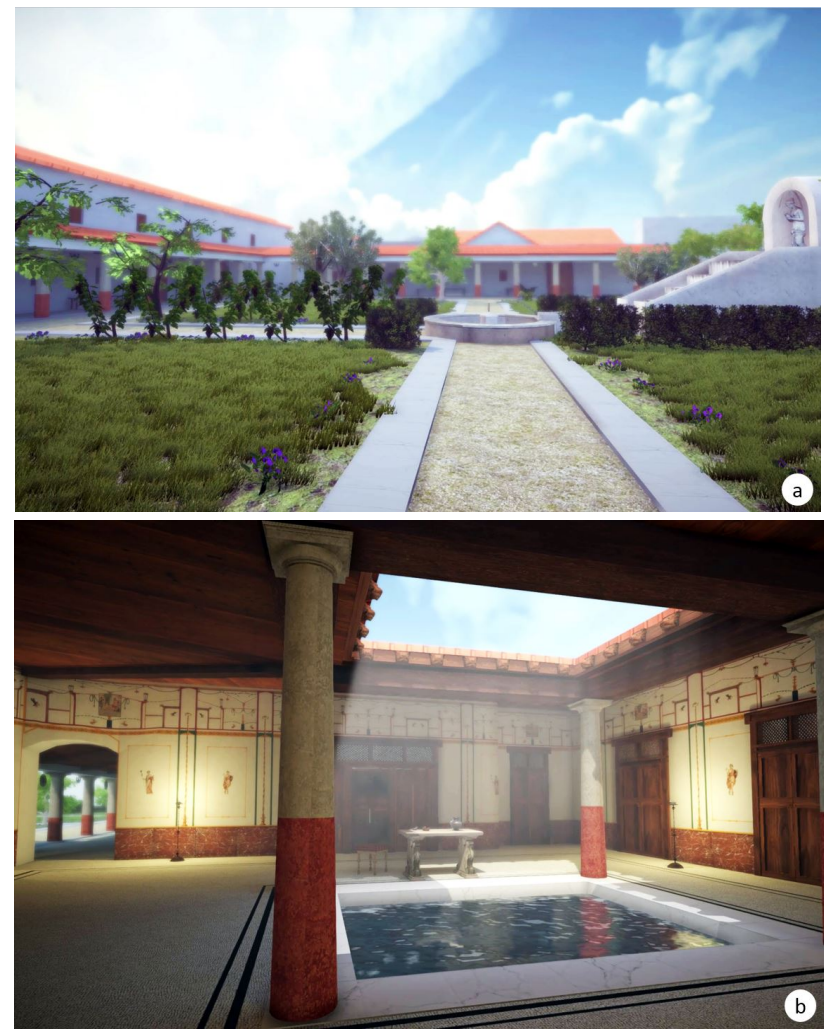




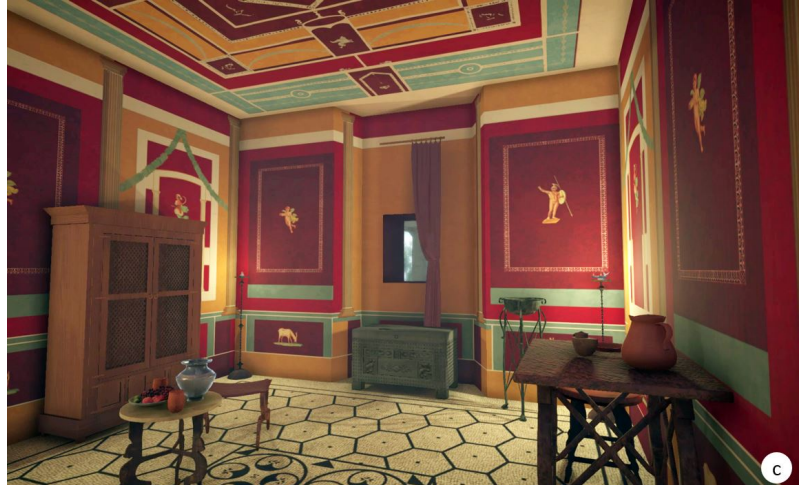

Figure 5. Different portions of the 3D hypothetical reconstruction of the Villa: garden (a), atrium with impluvium (b), room with pelte mosaic (c)

\subsection{0-degree videos}

The 360-degree videos use the classical film language and shot composition tools. With the exception of the first video, all of them are settled in the 3D hypothetical reconstruction of the complex of the "Villa con ingresso a protiro" and the actors are real people who look like Romans and who are of appropriate age for playing the characters in the stories (Rizvic et al. 2017). Furthermore, the visual styling of actors and their costumes were created in collaboration with the archaeologists to recreate the life accurately in the Roman period. These 360-degree videos have been developed according to a storytelling approach to catch the user's attention and enhance his/her experience while performing the virtual tour of the sunken archaeological site.

The storytelling has been conceived and written by Barbara Davidde Petriaggi and Roberto Petriaggi. In particular, it consists of six parts: the intro story has been settled in the Baiae remains on land (fig.6a) with a voice introducing the viewer with the city and its historical significance; the sculptor's workshop where four characters, i.e., the sculptor and his apprentice, the aristocrat and the slave, introduce themselves; the street with shops (fig.6b); the villa entrance; the room with mosaics (fig.6c); the atrium where the sculptor introduces his apprentice with the villa; and the discussion of the sculptor and the aristocrat about the statue design and price.
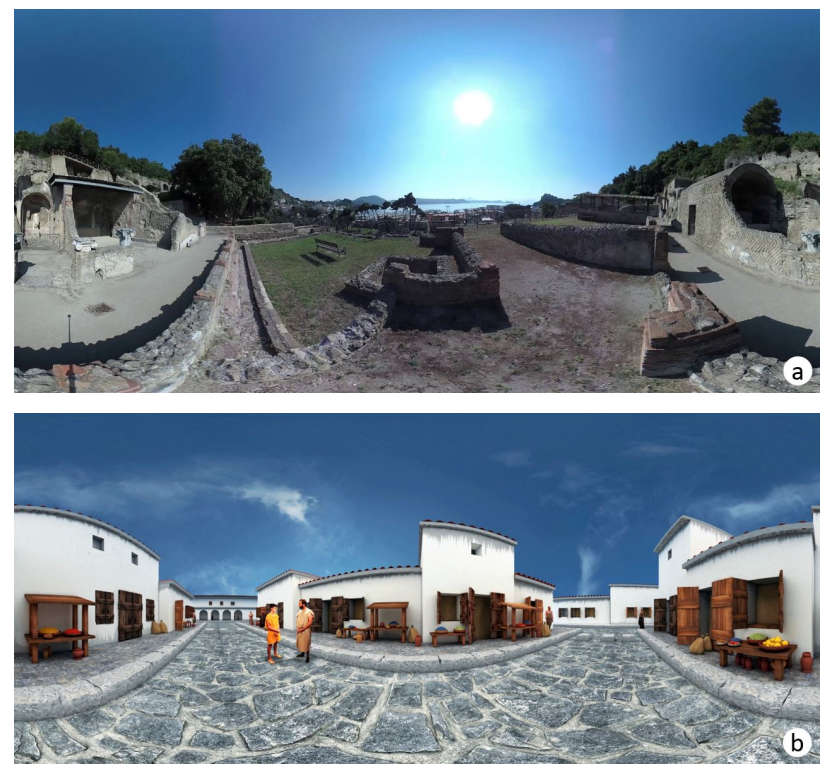

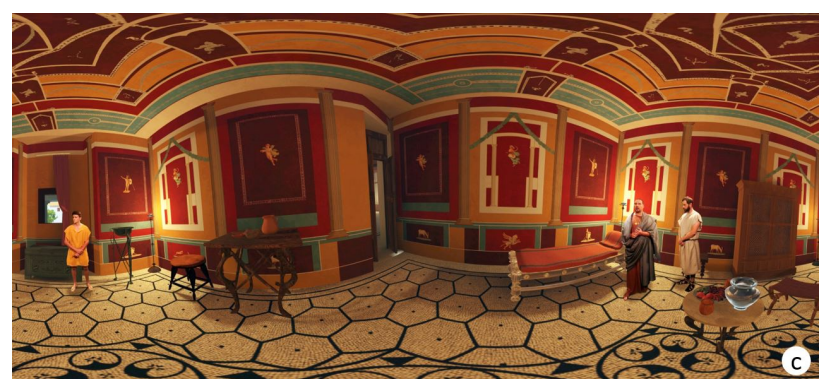

Figure 6.360-degree videos settled in: Baiae remains on land (a); the room with pelte mosaic (b); outdoor of the "Villa con impresso a protiro" (c)

\section{GAME DESIGN}

\subsection{VR storytelling}

The virtual tour of the sunken "Villa con ingresso a protiro" has been developed according to the best practices related to the gamification design process in order to maximize enjoyment and engagement by capturing the interest of the users. Indeed, it has been designed to raise users' knowledge and cultural awareness by providing them a faithful and realistic virtual replica of the actual underwater archaeological site that can be explored by means of an edutainment approach. The game logic presents three distinct elements: exploration, storytelling, and interaction. The exploration of the underwater archaeological site starts above the water surface. In order to make a more attractive and engaging experience, the terrestrial environment representing the coastline that overlooks the archaeological site has been added to the scene. It can be performed in two different modes that can be selected by the user: a free or guided tour. In the first mode, the user can dive freely in the archaeological area, and he/she is free to pick the desired POI or simply take an overview of the submerged area. When selected, each POI provides an audio description of the historicalarchaeological content related to the specific location. In the other case, the guided tour mode features a virtual dive buddy who guides the user during the exploration of the underwater archaeological site (fig.7). In particular, the virtual diver implements a logical follow-on of the POIs that, according to a storytelling approach, guide the user among the storytelling videos. Thanks to the virtual guide, users explore the area and play the videos in a precise order. When a POI is activated, the related full-screen 360-degree video is played. Users can also switch from the 3D representation of the Villa to the reconstruction of his ancient status by interacting with a special POI placed in the virtual scene. They can explore the original status of the structures, environments and even furnishings.

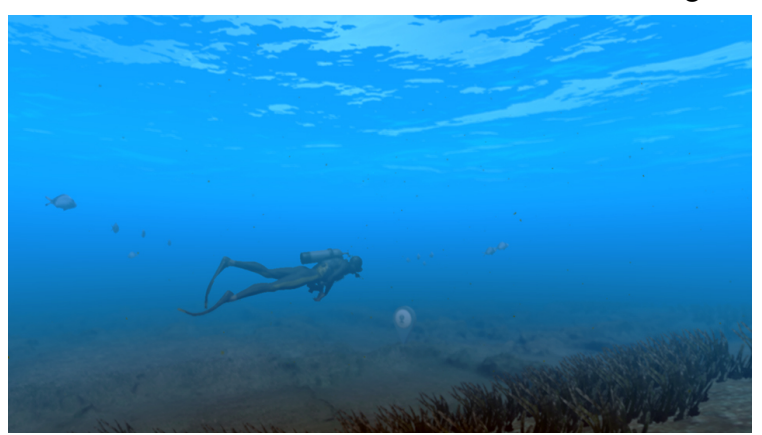

Figure 7. Scuba guide for the exploration of the underwater archaeological site 


\subsection{User interaction}

In order to provide to the users a fully immersive experience of the underwater archaeological site and to achieve the best results in terms of engaging (fig.8) the user interaction occurs by means of an HMD technology (Section 3.2) and one or two wireless controllers. When the user wears the HMD, he/she experiences the immersive virtual environment from the scuba diving viewpoint simulating a real diving session. The scenario that appears at the beginning of the virtual experience is above the water surface in the diving spot. Once he/she dives in the submerged virtual environment, depending by the abovementioned exploration modality chosen, i.e., free or guided tour, a directional 3D arrow or the virtual scuba guide shows the direction to reach the underwater archaeological area.

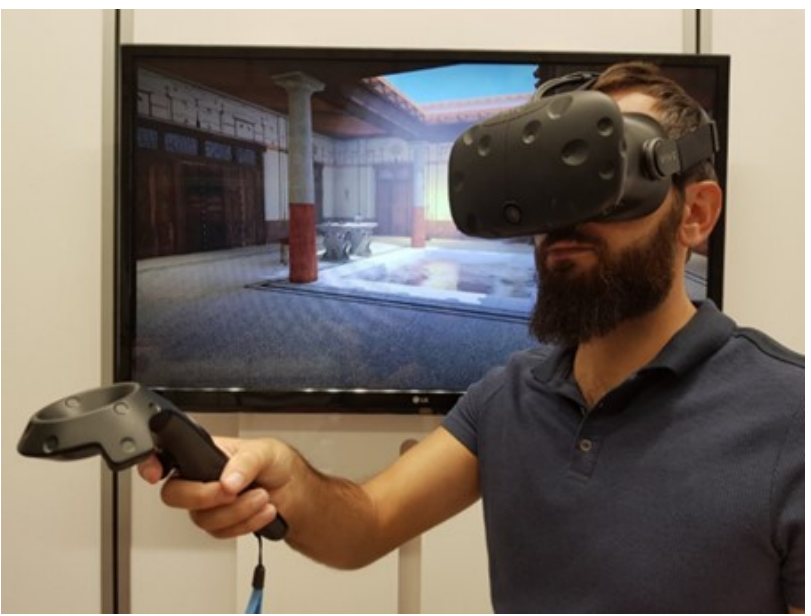

Figure 8. HMD technology for the immersive virtual diving system

The user explores the virtual environment by moving his/her head, and interacts with the POIs by means of the wireless handheld controllers (fig.9). Furthermore, through the input devices, the user instructs the software about the desired orientation and direction to follow for navigate within the submerged environment. During the navigation, the controllers are mainly used to explore the virtual underwater environment (to "swim" the user has to pull the hair trigger of the handheld controller while he/she is pointing in the direction he/she wants to go on). Nevertheless, when the user reaches a POI, this needs to be activated to get access to its multimedia contents. In the immersive environment, the POIs are enabled directly by the user, by pointing and selecting them by clicking on the touch button. In this case, the multimedia contents are displayed within the virtual scenario into a $3 \mathrm{D}$ frame using the other controller.

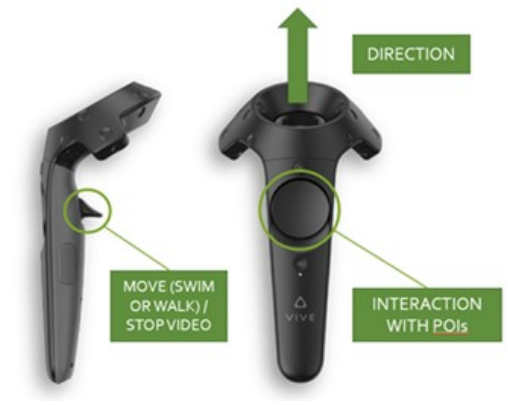

Figure 9. Interaction controller in VR application

\section{CONCLUSIONS}

The paper has presented the application of the technologies and methods developed in the i-MARECULTURE project for the virtual exploration of the complex of the "Villa con ingresso a protiro" located in the Marine Protected Area - Underwater Park of Baiae. In particular, the virtual tour described in the paper is an efficient instrument of communication and valorisation of the UCH because of its capability to allow users to explore an accurate and realistic 3D replica of the underwater archaeological site and to provide them a scientifically sound virtual reconstruction of the archaeological and architectural ruins.

Preliminary tests with the users have provided positive feedback about the usability and the engagement of the VR system. A formal assessment is currently in progress and will be published in future papers.

\section{ACKNOWLEDGEMENT}

This work has been supported by the i-MARECULTURE project that has received funding from the European Union's Horizon 2020 research and innovation programme under grant agreement No. 727153 .

\section{REFERENCES}

Chapman, P., Conte, G., Drap, P., Gambogi, P., Gauch, F., Hanke, K., Richards, J., 2006. Venus, virtual exploration of underwater sites. In: Proceeding of joint event CIPA/VAST/EG/Euro-Med.

Bruno, F., Lagudi, A., Gallo, A., Muzzupappa, M., Davidde Petriaggi, B., Passaro, S., 2015. 3D documentation of archaeological remains in the underwater park of Baiae. International Archives of the Photogrammetry, Remote Sensing \& Spatial Information Sciences. doi.org/10.5194/isprsarchivesXL-5-W5-41-2015.

Bruno, F., Lagudi, A., Muzzupappa, M., Lupia, M., Cario, G., Barbieri, L., Passaro, S., Saggiomo, R., 2016. Project VISAS Virtual and augmented exploitation of Submerged Archaeological Sites: overview and first results. Mar Technol Soc J (MTS) 50(4):119-129. doi.org/10.4031/MTSJ.50.4.4.

Bruno, F., Lagudi, A., Ritacco, G., Cejka, J., Kouril, P., Liarokapis, F., Agrafiotis, P., Skarlatos, D., Philpin-Briscoe, O., Poullis, E.C., 2017. Development and integration of digital technologies addressed to raise awareness and access to European underwater cultural heritage. An overview of the H2020 i-MARECULTURE project. In Proceedings of the MTS/IEEE Conference Oceans'17, Aberdeen, UK, 19-22 June 2017. doi.org/ 10.1109/OCEANSE.2017.8084984.

Bruno, F., Barbieri, L., Lagudi, A., Cozza, M., Cozza, A., Peluso, R., Muzzupappa, M., 2018. Virtual dives into the underwater archaeological treasures of South Italy. Virtual Reality, 22(2), 91-102. doi.org/10.1007/s10055-017-0318-z.

Davidde Petriaggi, B., 2017, Documentazione 3D per la conservazione del patrimonio archeologico sommerso: l'esperienza dell'ISCR, in Colombo A., Perzolla V. (a cura di), Le tecnologie digitali al servizio della conservazione, Atti del Convegno (Vicenza, 19 marzo 2016), Padova 27-31. 
Davidde Petriaggi, B., Petriaggi, R., Bruno, F., Lagudi, A., Peluso, R., Passaro, S., 2018. A digital reconstruction of the sunken "Villa con ingresso a protiro" in the underwater archaeological site of Baiae. In IOP Conference Series: Materials Science and Engineering (Vol. 364, No. 1, p. 012013). IOP Publishing. doi.org/10.1088/1757$899 X / 364 / 1 / 012013$.

iMareCulture project web-site: http://www.imareculture.eu/ (accessed on 1 March 2019).

Liarokapis, F., Kouril, P., Agrafiotis, P., Demesticha, S., Chmel1k, J., Skarlatos, D., 2017. 3D modelling and mapping for virtual exploitation of underwater archaeology assets. Int Arch Photogramm Remote Sens Spat Inf Sci XLII-2/W3:425-431. doi.org/10.5194/isprs-archives-XLII-2-W3-425-2017.

Mangeruga, M., Bruno, F., Cozza, M., Agrafiotis, P., Skarlatos, D., 2018. Guidelines for Underwater Image Enhancement Based on Benchmarking of Different Methods. Remote Sensing, 10(10), 1652. doi.org/10.3390/rs10101652.

Petriaggi, R., Mancinelli, R., 2004. An experimental conservation treatment on the mosaic floor and perimeter walls of room n. 1 of the so-called «Villa con ingresso a protiro» in the underwater archaeological park of Baia (Naples), in Archaeologia Maritima Mediterranea I, 109-126.

Skarlatos, D., Agrafiotis, P., Balogh, T., Bruno, F., Castro, F., Petriaggi, B.D., Demesticha, S., Doulamis, A., Drap, P., Georgopoulos, A., 2016. Project iMARECULTURE: Advanced VR, iMmersive serious games and augmented reality as tools to raise awareness and access to European underwater cultural heritage. In Proceedings of the International Conference on Cultural Heritage, Nicosia, Cyprus, 1-5 November 2016. doi.org/10.1007/978-3-319-48496-9_64.

Rizvic, S., Djapo, N., Alispahic, F., Hadzihalilovic, B., Cengic, F.F., Imamovic, A., Okanovic V, Boskovic, D., 2017. Guidelines for interactive digital storytelling presentations of cultural heritage. In 9th International Conference on Virtual Worlds and Games for Serious Applications (VS-Games), 253259. doi.org/10.1109/VS-GAMES.2017.8056610. 